\title{
Quantitative analysis of contrast-enhanced ultrasonography in acute radiation-induced liver injury: An animal model
}

\author{
JUN FENG $^{1}$, SHU-BO CHEN ${ }^{2}$, SHU-JUN WU ${ }^{1}$, PING SUN $^{3}$, TIAN-YOU XIN ${ }^{1}$ and YING-ZHEN CHEN ${ }^{1}$ \\ Departments of ${ }^{1}$ Ultrasound, ${ }^{2}$ Oncology and ${ }^{3}$ Pathology, The Second Hospital of Wuxi \\ Affiliated to Nanjing Medical University, Wuxi, Jiangsu 214002, P.R. China
}

Received July 15, 2015; Accepted September 2, 2015

DOI: $10.3892 /$ etm.2015.2764

\begin{abstract}
The aim of the present study was to examine and assess contrast-enhanced ultrasound in the early diagnosis of acute radiation-induced liver injury in a rat model. Sixty female rats were used, with 50 rats being utilized to produce an animal model of liver injury with a single dose of stereotactic X-ray irradiation of $20 \mathrm{~Gy}$. Ten rats from the injury group and 2 rats from the control group were randomly selected on days 3, 7, 14, 21 and 28, and examined by contrast-enhanced ultrasound and histopathology of liver specimens. The rats were divided into four groups: the normal control group, mild, moderate, and severe radioactive liver injury groups based on the histopathological examination results. Hepatic artery arriving time (HAAT) and hepatic vein arriving time (HVAT) were recorded, and hepatic artery to vein transit time (HA-HVTT) was calculated. The time-intensity curve of liver parenchyma, the time to peak (TTP) and peak intensity (PI) were also obtained. Significant differences were observed between liver injury and control groups for PI and HA-HVTT $(\mathrm{P}<0.05)$. PI and HA-HVTT were shorter in the severe liver injury group compared to the mild and moderate liver injury groups $(\mathrm{P}<0.05)$. Compared to the control group, higher TTP was recorded in all the liver injury groups $(\mathrm{P}<0.05)$, and the highest TTP level was observed in the severe liver injury group compared to the mild or moderate group $(\mathrm{P}<0.05)$. However, no significant difference was observed between the mild and moderate groups for PI, HA-HVTT and TTP. In conclusion, the results showed that contrast-enhanced ultrasonography is useful for an earlier diagnosis in a rat model of acute radiation-induced liver injury.
\end{abstract}

Correspondence to: Dr Shu-Bo Chen, Department of Oncology, The Second Hospital of Wuxi Affiliated to Nanjing Medical University, 68 Zhongshan Road, Chong'an, Wuxi, Jiangsu 214002, P.R. China

E-mail: drcsb2000@aliyun.com

Key words: ultrasonography, contrast media, radiation-induced liver injury

\section{Introduction}

In recent years, with the rapid development of radiotherapy technologies, three-dimensional conformal and intensity-modulated radiotherapy has been widely used for the treatment of primary hepatocellular carcinoma due to its efficacy to induce apoptosos or lose its infinite proliferation ability, so as to control tumor growth and make surrounding normal tissues and organs less affected by irradiation (1). However, radiation-induced liver injury following radiotherapy has become the major factor influencing the radiation tolerance dose (1). Liver is a type of late-responding tissue, and most of the earlier acute radiationinduced liver injuries can be repaired. Nonetheless, acute radiation-induced liver injuries may develop into sub-acute radiation-induced liver injury should clinically appropriate treatment not be administered, a phenomenon known as radiation-induced liver disease (RILD) (2). This disease is difficult to detect due to its occult clinical manifestations, but progresses until the failure of liver functions (2). Thus, earlier detection and diagnosis of acute radiation-induced liver injury is of great significance for restorative treatment. The importance of adjustment of the tumor radiotherapy dose has been previously reported (2). In the present study, we established a rat model of acute radiation-induced liver injury, and detected a relationship between quantitative analysis of contrast-enhanced ultrasound parameters and pathological parameters indicating severity of liver injury.

\section{Materials and methods}

Animals. Sixty female SD rats, weighing 180-200 g, aged 6 weeks were provided by the Experimental Animal Center of the Zhejiang Academy of Medical Sciences (Zhejiang, China). The rats were randomly divided into the control (10 rats) and experimental (50 rats) groups, and kept on the same normal diet throughout the experiment.

Acute radiation-induced liver injury model. The animals were initially anesthetized with $10 \%$ chloral hydrate $(300 \mathrm{mg} / \mathrm{kg})$ by intraperitoneal injection and were fixed in the supine position. The right lobe of the liver was located with B ultrasound and the radiation field was determined: the upper boundary was diaphragm, the lower boundary was $3 \mathrm{~cm}$ from the upper one, the medial boundary was right of the spine, but there was no 
lateral boundary. The radiation field was $\sim 3 \times 3 \mathrm{~cm}$ and was drawn on the surface. The Siemens PrimusM linear accelerator and $6 \mathrm{MV}$-X-ray source skin distance irradiation technique were used with a source skin distance $(\mathrm{SSD})$ of $80 \mathrm{~cm}$; irradiation depth, $2 \mathrm{~cm}$; irradiation dose rate, $200 \mathrm{cGy} / \mathrm{min}$; total dose of single radiation, $20 \mathrm{~Gy}$. Ten rats from the injury group and 2 rats from the control group were randomly selected on days 3, 7, 14, 21 and 28, and underwent liver contrast-enhanced ultrasound (CEUS). The animals were then sacrificed by decapitation and liver tissue was removed and dissected for the pathological examination.

\section{Ultrasonography examination}

i) Preparations prior to the examination. After fasting for $8 \mathrm{~h}$, the rats were anesthetized with $10 \%$ chloral hydrate (300 $\mathrm{mg} / \mathrm{kg}$ ) by intraperitoneal injection. Limbs were fixed in the supine position. The experimenter clamped the tail of the rat with two fingers to lead to caudal vein distention. Venipuncture was performed with a scalp acupuncture of 5 and a half. A venous channel was established and maintained.

ii) Instruments and methods. CEUS examination was performed using S2000 color ultrasonic diagnostic apparatus (Siemens Ultrasonography, Mountain View, CA, USA). The 9L4 probe was used for fundamental imaging at 7-9 Mhz and for the contrast-enhanced ultrasound imaging at $4 \mathrm{Mhz}$. The contrast-tuned imaging (cnTI) was built-in and quantitative analysis of contrast-enhanced ultrasound imaging software (Contrast Dynamics) was used. Ultrasonography contrast agent SonoVue (Bracco, Milan, Italy) was used. The diameter of ultrasonography contrast agent is far less than the untrasonic wavelength. It can form a new acoustic interface after entering the blood vessel with the help of the scattered signal of the micro bubbles, so as to produce a contrast effect. SonoVue was supplied as a lyophilized powder and reconstituted with $5 \mathrm{ml}$ of saline to form a homogeneous microbubble suspension with sulfur hexafluoride encapsulated in phospholipids. The mechanical index was 0.10 (the mechanical parameters of the experimental conditions showing consistency and repeatability) and the volume of contrast agent injected was $0.06 \mathrm{ml}$. Microbubble suspension was injected through the caudal vein. Each injection was followed by a $5 \mathrm{ml}$ saline flush. Liver transverse section showing liver parenchyma, the inferior vena cava, the abdominal aorta, the portal vein and other large vessels was considered. CEUS was performed immediately after contrast medium injection and lasted for $90 \mathrm{sec}$. The results were stored in a hard disk for subsequent offline analysis.

Parameter measurement. Hepatic parenchyma of region of interest (ROI) with the principle of consistent depth and avoidance of large vessels were selected. The time-intensity curve (TIC) of liver parenchyma was drawn automatically. The time to peak (TTP) and peak intensity (PI) were also analyzed with quantitative analysis of contrast-enhanced ultrasound imaging software (Fig. 1). Arriving time (AT) was defined as the time of the emergence of the first contrast agent microbubble in the vessel. Hepatic artery arriving time (HAAT) and hepatic vein arriving time (HVAT) were recorded, and hepatic artery to vein transit time (HA-HVTT) was the difference of the two times.

Pathological examination. The pathological sections were prepared with liver tissue of irradiation region in the right lobe of the liver in rats and were read using the double-blind method. Pathology grouping criteria adhered to were: i) Normal control group: normal liver cells, hepatic lobule structure integrity, and hepatic cord arranged in neat rows; ii) mild liver injury group: slight swelling of liver cells, fatty and/or hydropic degeneration, no liver cell necrosis and hemorrhage, and mild hepatic sinus dilation; iii) moderate liver injury group: moderate swelling of liver cells, fatty and/or hydropic degeneration, punctate necrosis of liver cells or scattered bleeding points, inflammatory cell infiltration in the portal area, and centrilobular vein and hepatic sinus dilation; and iv) severe liver injury group: diffused swelling and degeneration of liver cells, liver cell necrosis and hemorrhage, an apparent increase in inflammatory cells in the portal area, and veno-occlusive disease (VOD) of hepatic sinus and centrilobular veins (Fig. 2).

Statistical analysis. Continuous variables relevant to the normal distribution are shown as mean \pm SD. The univariate analysis of variance was conducted to compare differences between groups. The LSD test was used to compare differences between any two groups. The data were analyzed using SPSS, version 18.0 (IBM, Armonk, NY, USA). $\alpha=0.05$ was used for the statistical comparisons. $\mathrm{P}<0.05$ was considered to indicate a statistically significant result.

\section{Results}

Pathological results. An acute radiation-induced liver injury model was successfully established in rats and complete information from 60 animals (including 10 controls) was obtained. Liver biopsy results showed 10 cases of control, and 50 cases of radioactive liver injury (16 cases of mild, 22 cases of moderate, and 12 cases of severe liver injury).

\section{Ultrasonography results}

i) Significant differences were observed for PI $(\mathrm{P}<0.05)$ between the control and liver injury groups. Compared to the mild and moderate liver injury groups, PI was apparently shorter in the severe liver injury group $(\mathrm{P}<0.05)$, with no significant difference being observed between the mild and moderate groups.

ii) TTP was significantly different between the liver injury and control groups $(\mathrm{P}<0.05)$. TTP was much longer in the severe liver injury group compared to the mild and moderate liver injury groups $(\mathrm{P}<0.05)$, while no significant differences were observed between the mild and moderate groups.

iii) Compared to the control group, HA-HVTT in the moderate and severe liver injury groups was significantly higher $(\mathrm{P}<0.05)$, whereas no significant differences were detected between the mild and control groups.

There were significant differences among the indicators between groups (Tables I and II). The aggravation of radiation-induced liver injury was accompanied by the trends of decreased peak strength, prolonged peak time and reduced HA-HVTT.

\section{Discussion}

Since the liver is highly sensitive to radiation, the radiation-induced liver injury caused by radiotherapy is difficult to 


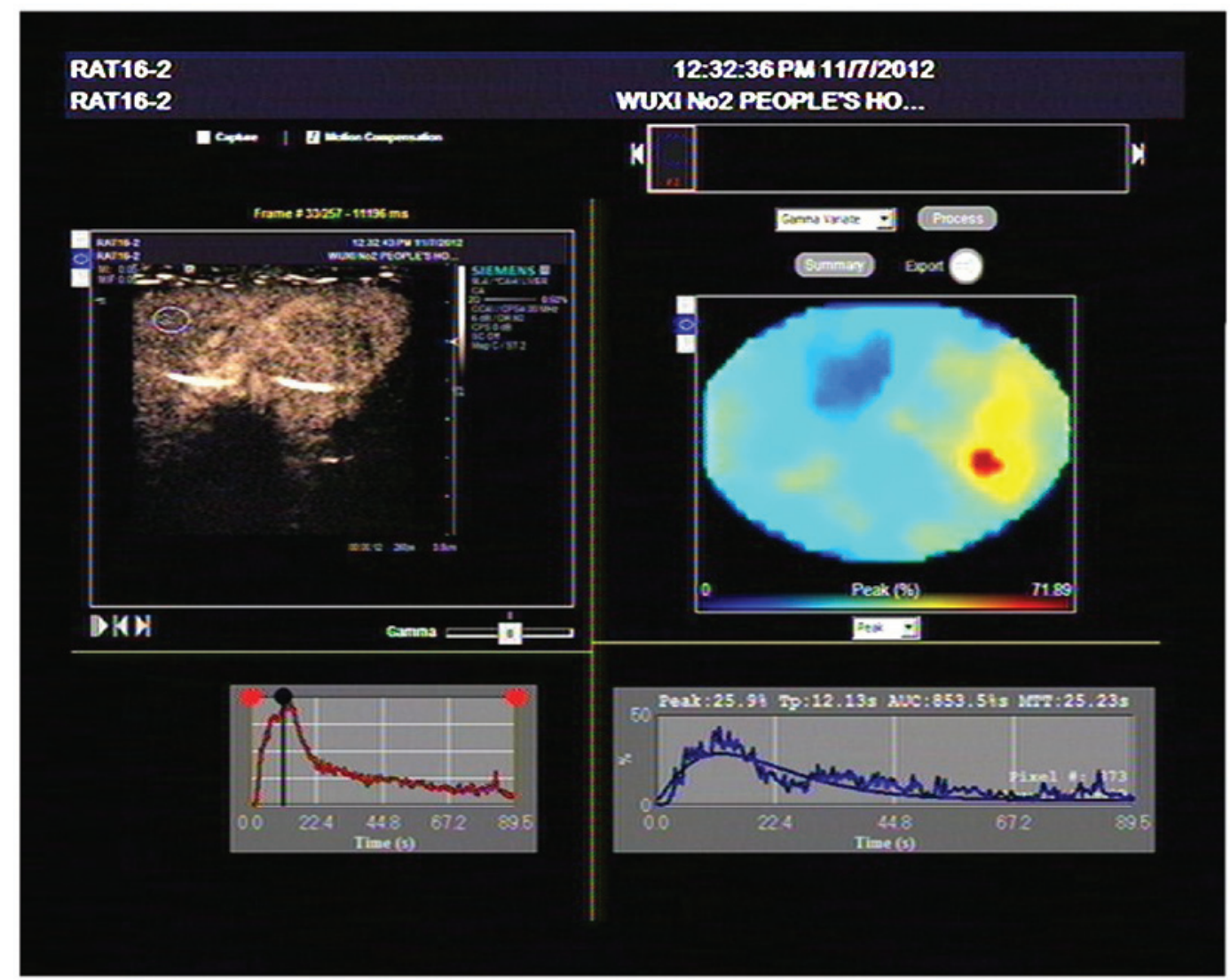

Figure 1. Rat contrast-enhanced ultrasound. The upper left panel shows the oval cross section of rat liver. The white box (upper right panel) is for the region of interest (ROI). The red curve (lower left panel) is for the whole liver contrast-enhanced ultrasound curve. The lower right panels show the liver parenchyma time intensity curve generated by the analysis software of contrast-enhanced ultrasound and the experimental data.
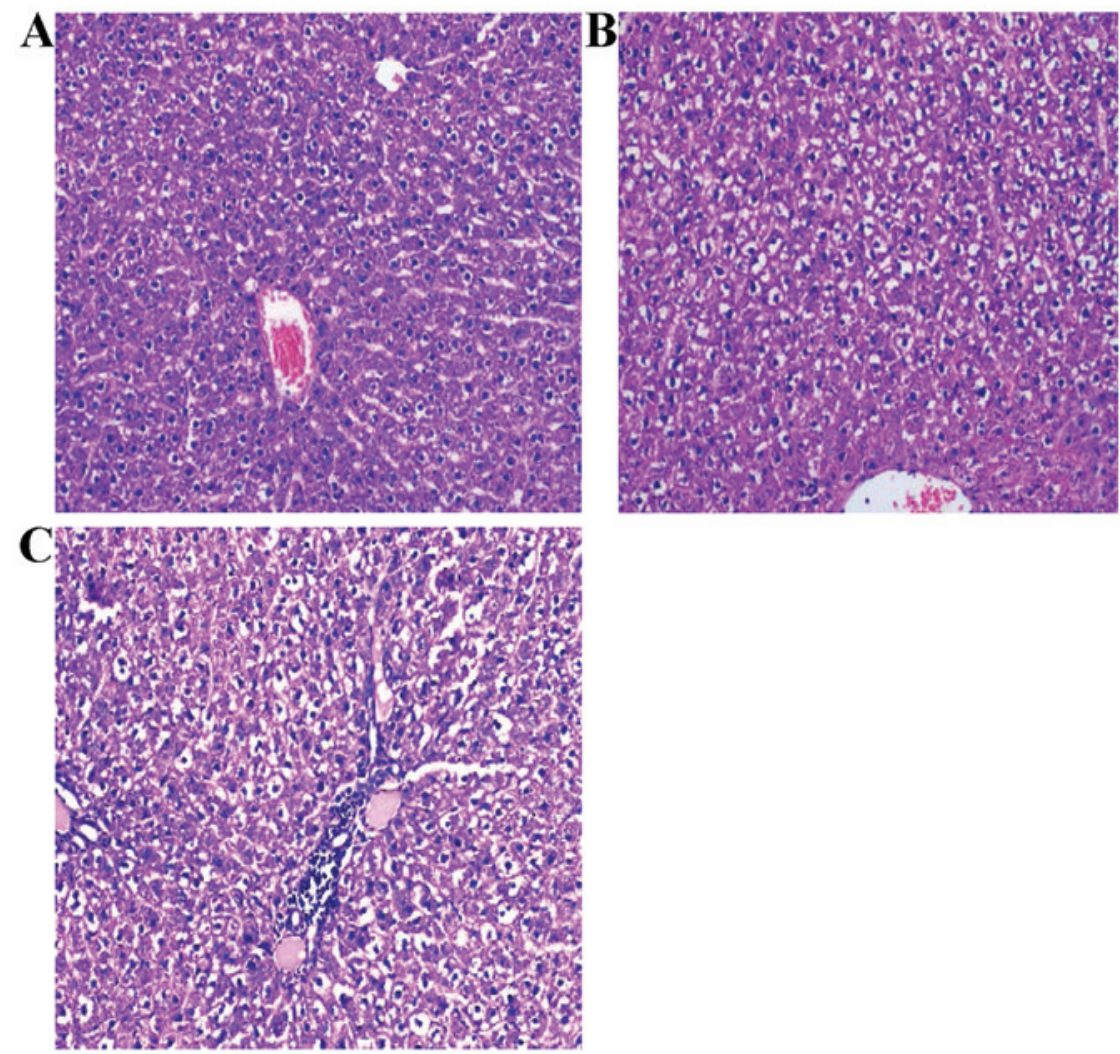

Figure 2. Pathology section of the rat acute radiation-induced liver injury (H\&E, x100). (A) Mild liver injury with slight edema of liver cells. (B) Middle hepatic lesions with hydropic degeneration, and punctate hepatocellular bleeding points. (C) Severe liver injury with diffuse hydropic degeneration, liver cell necrosis and hemorrhage. Inflammatory cells increased in the portal area with congestion in the hepatic sinusoid. 
Table I. Comparison of contrast-enhanced ultrasound parameter of the liver radiation-induced injury in different groups.

\begin{tabular}{lccl}
\hline Groups & Cases & PI (dB) & TPP (sec) \\
\hline Normal control group & 10 & $57.98 \pm 8.55$ & $17.26 \pm 7.43$ \\
Mild injury group & 16 & $41.82 \pm 8.69^{\mathrm{a}}$ & $26.49 \pm 6.8^{\mathrm{a}}$ \\
Moderate injury group & 22 & $35.27 \pm 14.28^{\mathrm{a}}$ & $28.64 \pm 10.77^{\mathrm{a}}$ \\
Severe injury group & 12 & $26.35 \pm 5.52^{\mathrm{a}-\mathrm{c}}$ & $41.51 \pm 11.23^{\mathrm{a}-\mathrm{c}}$ \\
F-value & 17.355 & 12.488 & \\
P-value & $<0.001$ & $<0.001$ & \\
\hline
\end{tabular}

Compared with the normal control group, ${ }^{\mathrm{a}} \mathrm{P}<0.05$. Compared with the mild liver injury group, ${ }^{b} \mathrm{P}<0.05$. Compared with the moderate liver injury group, ${ }^{\mathrm{C}}<0.05$. PI, peak intensity; TPP, time to peak.

avoid, but has a high degree of reversibility. RILD occurred 4 weeks after radiotherapy and based on the sub-acute damage of liver function is known as local radiation-induced hepatic injury. Previous findings performed on acute radiation-induced liver injury in animal models differ slightly in the radiation range, radiation dose, and inspection method (3). Preliminary results led to selection of the right lobe of the liver as the irradiation target area, and was determined as: the upper boundary was diaphragm, the lower boundary was $3 \mathrm{~cm}$ from the upper one, the medial boundary was right of the spine, and there was no lateral boundary. The radiation field was $\sim 3 \times 3 \mathrm{~cm}$ and was drawn on the surface. Single dose X-ray stereotactic irradiation was $20 \mathrm{~Gy}$. The observation time was 1-28 days following irradiation.

VOD is a vein occlusion type of disease which is the pathological manifestation of radiation-induced liver injury, characterized by congestion in the central area of the hepatic lobule and necrosis of the central area in impaired hepatic sinus under a microscope. The pathological change is a dynamic process, initially involving a cell dysfunction phase, followed by a radioactive hepatitis phase and subsequently a liver fibrosis and cirrhosis phase $(3,4)$. It has been previously shown that the specific functional abnormality occurs at a very early stage after irradiation (mostly in the first month) and is known as acute radiation-induced liver injury (5). The main manifestations include dilatation of the central venous and sinus in hepatic lobules as congestion is the main response to irradiation, and endothelial cell injury throughout the process, which is the basis of hepatic veno-occlusive lesions. Hepatic sinus congestion after endothelial cell injury and inflammatory cell infiltration is the main lesion of early radiation-induced hepatic injury. The degeneration and necrosis of liver cells are observed two weeks after irradiation, mainly including eosinophilic necrosis and punctate necrosis.

The blood supply to normal liver is mainly derived from the hepatic artery and portal vein, and the terminal branches of hepatic arterioles and portal vein converge with hepatic vein in sinus hepaticus (6). The arteriovenous direct path is closed under normal circumstances; however, it opens in some liver diseases manifested as hepatic hemodynamic changes (6). The microbubble ultrasound contrast agent currently used possesses hemodynamic characteristics that are similar to red
Table II. Comparison of HA-HVTT of radiation-induced liver injury in each group.

\begin{tabular}{lcc}
\hline Group & Cases & HA-HVTT $(\mathrm{sec})$ \\
\hline Normal control group & 10 & $8.12 \pm 0.75$ \\
Mild injury group & 16 & $7.5 \pm 0.94$ \\
Moderate injury group & 22 & $7.11 \pm 0.89^{\mathrm{a}}$ \\
Severe injury group & 12 & $5.78 \pm 0.5^{\mathrm{a}-\mathrm{c}}$ \\
F-value & 16.757 & \\
P-value & $<0.001$ & \\
\hline
\end{tabular}

Compared with the normal control group, ${ }^{a} \mathrm{P}<0.05$. Compared with the mild liver injury group, ${ }^{b} \mathrm{P}<0.05$. Compared with the moderate liver injury group, ${ }^{\mathrm{c}} \mathrm{P}<0.05$. HA-HVTT, hepatic artery to vein transit time.

blood cells, and can filter through pulmonary circulation to reach microvascular organs and lesions allowing images to be captured (6). The ultrasound contrast agent can evaluate the perfusion of the organs and tumor from the perspective of microcirculation, and reflect the changes of organic hemodynamics and eventually contribute to the understanding of organ function (7).

The present study indicates that the hepatic hemodynamic changes have important effects on the liver blood perfusion in the process of the formation and development of acute radiation-induced liver injury. The TIC curve of the liver parenchyma contrast-enhanced ultrasound showed that with the aggravation of the liver injury, PI continued to decrease and TP was gradually extended with statistically significant differences. Possible reasons for this finding include, fat degeneration and swelling of liver cells occurring in the early phase of acute radiation-induced liver injury may lead to compression and degeneration of the hepatic duct structure and an increase in the resistance, resulting in the reduction of liver parenchyma blood perfusion $(6,7)$. Additionally, with the development of hepatic injury, liver cell necrosis, inflammatory cell infiltration and hepatic sinusoid capillarization occurred, which eventually leads to the formation of blood stasis in the hepatic sinusoids, the increase of sinus pressure and further formation of intrahepatic shunt, resulting in a blood flow reduction in the liver parenchyma (8). Furthermore, formation of fiber spacing due to injury of the oppressed sub-lobular vein, central vein and hepatic venous sinus leads to disturbance of the recovery of the portal vein, an increase in portal vein pressure, and reduced liver perfusion, resulting in a decrease in the extension of PI and TP. The abovementioned three changes are increased with aggravation of liver injury.

In the present study, HA-HVTT was gradually reduced along with the severity of liver damage, and these differences were statistically significant in the inter-group comparison. Possible reasons for this finding include the fact that in the early phase of acute liver injury, liver sinusoidal endothelial cells are impaired; endothelial fenestrae are reduced or gradually dissipate; and a continuous structure-like capillary endothelium.is formed beneath the endothelial, which leads to the formation of hepatic sinusoid capillarization. These changes form the exchange barrier between substances in liver 
cells and oxygen and eventually lead to liver cell atrophy and hepatic sinusoidal collapse. Blood flow in hepatic sinusoid gradually bypasses the damaged liver tissue and enters the hepatic venous system directly. As a result, blood circulation is accelerated in the liver and HA-HVTT is shortened (9-11). In the process of liver injury, a variety of neurotransmitters responding to the course such as histamine, serotonin, and vasoactive factors, including vascular endothelium-derived relaxing factor, cause damage to the hepatic sinusoids as well as hepatic vein system and the disturbance of liver microcirculation. Therefore, the hepatic blood flow at a high level of circulation, which accelerates the distribution of contrast agent microbubbles in vivo (12-14). Additionally, with the aggravation of liver injury, the formation of cytokines containing transforming growth factor- $\beta 1$ (TGF- $\beta 1$ ), platelet-derived growth factor (PDGF), and connective tissue growth factor (CTGF), promotes collagen synthesis by hepatic stellate cells (HSCs) and accelerates the transformation from HSCs to fibroblast cells. Proliferous fibroblasts and their connection with fiber bundles around them form a fibrous septum, in which the blood vessels form rami communicantes among hepatic arteries, portal veins and hepatic veins. Blood bypasses due to rami communicantes, resulting in enhancement of HVAT and shortening of HA-HVTT (8). However, radiation-induced liver injury is a gradual process, in which the expression of cytokines is not obvious in the early stages and gradually increases after 2 weeks. No significant differences were observed in the mild and control groups $(\mathrm{P}>0.05)$. Thus, HA-HVTT as a sensitive index to evaluate the severity of liver fibrosis is important in the early diagnosis of moderate and severe acute radiation-induced liver injury.

Radiation-induced liver injury is a progressive process, and early detection and early intervention in the acute period is of great significance to the recovery treatment of the liver. Contrast-enhanced ultrasound as a non-invasive, simple and highly repetitive examination can more intuitively reflect the changes of the microvascular circulation dynamics. The circulatory physiology of the contrast agent in liver is analyzed to identify the sensitive parameters applied in the quantitative diagnosis of acute radiation-induced liver injury. The present findings have shown that in the quantitative analysis of contrast-enhanced ultrasonography, PI, TP and HA-HVTT may be used as important reference indices for the early diagnosis of acute radiation-induced liver injury.

\section{Acknowledgements}

This study was supported by the Science and Technology Development Foundation of Nanjing Medical University (no. 2011NJMU1234) and the Medical Science and Technology Development Fund Management Center of Wuxi Hospital (no. YGM1126).

\section{References}

1. Rahbari NN, Mehrabi A, Mollberg NM, Müller SA, Koch M, Büchler MW and Weitz J: Hepatocellular carcinoma: Current management and perspectives for the future. Ann Surg 253: 453-469, 2011

2. Bujold A and Dawson LA: Stereotactic radiation therapy and selective internal radiation therapy for hepatocellular carcinoma. Cancer Radiother 15: 54-63, 2011.

3. Peng RY, Wang DW, Xu ZH, et al: Dynamic observation of the process of radiation-induced liver fibrosis. Chin J Radiol Med Prot 14: 243-245, 1994 (In Chinese).

4. Fajardo LF: The pathology of ionizing radiation as defined by morphologic patterns. Acta Oncol 44: 13-22, 2005.

5. Liang SX, Zhu XD, Lu HJ, Pan CY, Li FX, Huang QF, Wang AY, Chen L, Fu XL and Jiang GL: Hypofractionated three-dimensional conformal radiation therapy for primary liver carcinoma. Cancer 103: 2181-2188, 2005.

6. Li N, Ding H, Lin XY, Fan PL, Xu C and Wang WP: Correlations between quantitative parameters of contrast-enhanced ultrasonography and histopathologic stage of liver fibrosis. Chin J Ultrason 18: 942-945, 2009 (In Chinese).

7. Zhang L, Duan YY,Zhang Y, et al: Primary research on diagnosis of liver fibrosis stage by perfusion dynamics of ultrasound contrast agent. Chin J Ultrason 16: 616-619, 2007 (In Chinese).

8. Jie LM, Guo QY, Liu X, et al: The experimental study on the evaluation of liver fibrosis using contrast enhanced sonography. Chin J Ultrason 17: 809-812, 2008 (In Chinese).

9. Shen JK, Jiang Z, Zhou J, et al: Experimental study on the early effects of radiation induced liver injury: the number and the function evaluation of Kupffer cells. Chin J Radiol Med Prot 6: 553, 2004 (In Chinese).

10. Zhou YX: The Modern Diagnosis and Treatment of Liver Cirrhosis. People's Medical Publishing House, Beijing, p14, 2000.

11. Lu X, Xu GF, Chen WH, et al: The dynamic changes of hepatic sinusoid capillarization during the formation of hepatic fibrosis in rats. Shi Jie Hua Ren Xiao Hua Za Zhi 8: 1415-1416, 2000 (In Chinese).

12. Li R and Hua X: Experimental study on the diagnostic value of the appearing time of ultrasound contrast agent in hepatic vein in early liver cirrhosis. Chongqing Med J 33: 1684-1685, 2004.

13. Wang CP and Han J: Cellular and molecular mechanism of portal hypertension. Infect Dis Info 18: 117-119, 2005.

14. Tan XQ, Qian LX,Zhang XL, et al: Contrast-enhanced ultrasound in diagnosis of early hepatic cirrhosis. Chin J Ultrason 17: 1048-1050, 2008 (In Chinese). 